\title{
Quaderni
}

QUADERN I Communication, technologies, pouvoir

81 | Printemps 2013

\section{L'humain médicament}

\section{Les médicaments de thérapie innovante : quelles spécificités en droit pharmaceutique?}

Advanced therapy medicinal products : which specific features in pharmaceutical law?

\section{Florence Taboulet}

\section{(2) OpenEdition}

\section{Journals}

Édition électronique

URL : http://journals.openedition.org/quaderni/702

DOI : 10.4000/quaderni.702

ISSN : 2105-2956

Éditeur

Les éditions de la Maison des sciences de l'Homme

Édition imprimée

Date de publication : 5 juin 2013

Pagination : 15-27

\section{Référence électronique}

Florence Taboulet, « Les médicaments de thérapie innovante : quelles spécificités en droit

pharmaceutique? », Quaderni [En ligne], 81 | Printemps 2013, mis en ligne le 05 juin 2015, consulté le

19 avril 2019. URL : http://journals.openedition.org/quaderni/702 ; DOI : 10.4000/quaderni.702 


\section{$D$ ossier}

\section{Les médicaments}

de thérapie innovante : quelles spécificités en droit pharmaceutique?

\section{Florence Taboulet}

Professeur Droit pharmaceutique et Économie de la santé Inserm, UMR U 1207, Université de Toulouse, Paul Sabatier - Toulouse III
Le droit pharmaceutique constitue l'une des trois branches du droit de la santé, à côté du droit médical et du droit hospitalier. Il s'est construit autour de la notion de médicament, ce qui l'amène aujourd'hui à se complexifier davantage ${ }^{1}$. Il intègre de plus en plus de ramifications puisque le nombre de catégories des médicaments, tant en droit de l'Union européenne (UE) qu'en droit français, ne fait que s'accroître, en y associant à chaque fois un nouveau lot de règles particulières. Cependant, ces définitions, ces classifications et les normes correspondantes ne sont pas nécessairement inscrites dans les législations nationales ou européennes comme dans le marbre. Les flous entourant les statuts juridiques sont particulièrement présents au niveau des dénominations des produits et préparations de thérapie génique et cellulaire et de leurs définitions. Si l'on croise les dimensions longitudinales et comparatives, la difficulté à qualifier un produit donné, à un moment donné et à identifier les règles qui s'y rapportent est extrême, puisque le législateur français avait devancé les initiatives communautaires en la matière. Alors que les premières définitions ont été introduites dans le code français de la santé publique (CSP) dès $1996^{2}$, puis modifiées en $2004^{3}$, c'est la directive 2001/83/CE qui encadre de façon claire et précise les produits destinés aux thérapies géniques et cellulaires somatiques au niveau européen. Les règles relatives à l'autorisation, la surveillance et la pharmacovigilance, ont été ensuite considérablement modifiées et étoffées en $2007^{4}$ avec le règlement relatif aux médicaments de thérapie innovante (MTI) qui regroupe les médicaments de thérapie génique, de thérapie cellulaire et une nouvelle catégorie, les médicaments issus de l'ingénierie cellulaire ou tissulaire. Pour chacune de ces trois catégories, 
un ou plusieurs dispositifs médicaux peuvent être associés. On les qualifie de médicaments combinés de thérapie innovante.

L'ensemble des produits et préparations de thérapie génique et cellulaire soulève des questions au cœur du droit pharmaceutique. La plupart de ces produits sont dorénavant appréhendés comme des médicaments, mais d'autres, comme les produits cellulaires ou tissulaires à finalité thérapeutique, ne le sont pas. La juxtaposition de ces multiples statuts devient un dédale sinon inintelligible, au moins extraordinairement compliqué. Le phénomène n'est pas nouveau car l'arsenal thérapeutique ne constitue pas, sur le plan juridique, un bloc monolithique en raison de la multiplication du nombre de spécialités pharmaceutiques. Les professionnels du secteur sont dès lors habitués à identifier des catégories particulières issues des classifications fondées sur le mode de préparation $^{5}$, sur la composition ${ }^{6}$ ou encore sur les modalités de prescription et de dispensation ${ }^{7}$. La plupart de ces catégories ont pour source le droit de l'Union européenne et ont été transposées en droit national ; mais quelques-unes proviennent du droit français et figurent soit dans le CSP, soit dans le code de la sécurité sociale (CSS), avec alors une dimension principalement économique.

La création et les modifications successives de ces nouvelles catégories de MTI doivent-elles être considérées comme une simple énième addition, banale, à la longue liste des définitions et des régimes particuliers de médicaments? Au contraire, ces MTI et leurs nouveaux « produitsfrontières » constituent-ils une innovation de telle sorte que des règles spécifiques doivent être mises en œuvre ? Y a-t-il continuité avec le régime de droit commun du médicament, ou peut-on identifier des ruptures significatives par rapport aux révisions engendrées par les autres spécialités pharmaceutiques?

Les spécificités relatives à ces produits faisant l'objet de cet article renvoient à un immense champ de promesses thérapeutiques, indissociables d'un changement d'échelle d'intervention sur le biologique puisque les MTI concernent la manipulation de la structure du vivant. Ces remaniements juridiques ne sont pas en définitive si originaux (1), alors que les spécificités au regard du corpus du droit pharmaceutique passent souvent inaperçues (2). Les sources originelles $\mathrm{du}$ droit pharmaceutique français étant majoritairement européennes, notre démarche s'appuie à la fois sur le droit de l'Union européenne et le droit français.

\section{Les spécificités discutables}

\section{Une réelle innovation : sur tous les plans?}

Le législateur européen a regroupé en 2007 les MTI au sein d'un ensemble nommé médicaments de thérapie innovante (Advanced therapy medicinal products).

Le premier considérant du règlement stipule : « Des progrès scientifiques récents en biotechnologie cellulaire et moléculaire ont conduit à la mise au point de thérapies innovantes, telles que la thérapie génique, la thérapie cellulaire somatique ou l'ingénierie tissulaire. Cette discipline naissante, la biomédecine, offre de nouvelles possibilités de traitement des maladies et des dysfonctionnements du corps humain. ». 
Deux éléments sont mis en avant : 1) le mode de fabrication de ces médicaments, rendu possible grâce à l'essor des biotechnologies, 2) les avancées thérapeutiques escomptées pour les patients. Nous analyserons successivement «ce quelque chose de nouveau » et son corollaire attendu, «ce quelque chose de meilleur ».

À quoi fait référence l'innovation? Puisque les sciences et les technologies se structurent de manière continue, il est bien difficile de dater l'émergence de l'application des connaissances de biologie moléculaire et de génétique sur la structure même du vivant humain. La définition du médicament biologique a été introduite dans le CSP en 2007 par une ordonnance qui transposait des dispositions communautaires : " tout médicament dont la substance active est produite à partir d'une source biologique ou en est extraite et dont la caractérisation et la détermination de la qualité nécessitent une combinaison d'essais physiques, chimiques et biologiques ainsi que la connaissance de son procédé de fabrication et de son contrôle. ${ }^{8}$. De fait, les parts en valeur de ces biothérapies dans le marché pharmaceutique hospitalier et dans le marché pharmaceutique officinal, ne cessent de croître. Cependant, il n'a pas fallu attendre le $\mathrm{XXI}^{\mathrm{e}}$ siècle pour que le vivant, d'origine végétale ou d'origine animale, mais aussi d'origine humaine soit utilisé pour réparer le vivant humain. La longue histoire des transplantations et des greffes et l'histoire plus récente de la transfusion sanguine, ou de la procréation médicalement assistée en sont des illustrations.

Au sein de ce vaste ensemble des biothérapies, les MTI présentent quelques spécificités. À la différence des médicaments classiques, y compris ceux d'origine humaine comme les dérivés hormonaux, le produit n'est plus administré à l'homme par voie locale, à travers un orifice du corps humain, per os, ou encore par voie parentérale. Il s'agit au contraire d'un traitement invasif qui est habituellement associé à la chirurgie ${ }^{9}$ et qui devient alors, comme pour beaucoup de dispositifs médicaux implantables, un traitement opérateur-dépendant. Ce qui est également nouveau, c'est la taille de la cible : cellule ou biomolécule ; dernier maillon dans la maîtrise de l'infiniment petit, de la structure intime des composants du corps humain. Aussi, en termes de connaissances exploitées et de mode d'action, la nouveauté s'inscrit dans la continuité des sciences et techniques biomédicales de la fin du XX $\mathrm{XX}^{\mathrm{e}}$ siècle. Il n'y a pas de différence de nature, mais de degré dans l'innovation liée aux MTI.

« Ce quelque chose de nouveau » est-il toujours accompagné de « ce quelque chose de meilleur»? Le bénéfice des thérapeutiques liées aux MTI, tant espéré par les patients, est-il significatif ? S'agit-il, aux yeux du clinicien et aux yeux de l'usager d'une innovation radicale ou d'une innovation incrémentale ? La dénomination " thérapie innovante " véhicule en soi des promesses de rupture. Cet usage sémantique n'est pas rattaché à un discours promotionnel de la firme qui revendique et met en avant ce label. Il s'agit du terme juridique associé à ces médicaments. L'idée première est celle d'une révolution biologique et d'un lot de guérisons spectaculaires pour des maladies jusque là dépourvues de traitement. C'est bien sûr ce qui est escompté. Mais le terme d'innovation 
est équivoque ; les critères de jugement du biologiste, de l'expert en génie génétique ou en propriété intellectuelle, du galéniste, ou du pharmacologue ne sont pas les mêmes que ceux du médecin ou du malade. La nouveauté ne se traduit pas toujours par la supériorité clinique ; Hippocrate le signalait déjà : « Le traitement nouveau dont on ne sait pas encore s'il est utile, est loué plus que le traitement habituel dont on est certain qu'il est utile. Les choses étranges le sont plus que les choses évidentes. ${ }^{10}$.

La meilleure illustration de cette dualité est apportée en France par l'exemple du jugement de la Commission de la transparence, chargée d'évaluer l'intérêt thérapeutique du ChondroCelect ${ }^{\circledR}{ }^{11}$, premier médicament de thérapie cellulaire ayant reçu de la Commission européenne une autorisation de mise sur le marché (AMM). Cette spécialité est indiquée dans la réparation des lésions cartilagineuses localisées et symptomatiques des condyles fémoraux du genou, chez l'adulte. On peut lire dans cet avis ${ }^{12}$ les conclusions suivantes : "Bien que la Commission considère qu'il s'agisse d'une biotechnologie innovante, le service médical rendu doit être provisoirement considéré comme insuffisant en l'état actuel des données, pour justifier sa prise en charge. ». Cette appréciation a arrêté le processus d'évaluation en vue de l'inscription du produit sur la liste des médicaments pris en charge par l'Assurance maladie, si bien que l'indicateur de mesure du progrès thérapeutique utilisé en France, l'amélioration du service médical rendu, n'a en l'occurrence même pas été quantifié. L'inscription du produit sur la liste a donc été refusée ${ }^{13}$, de telle sorte que son utilisation ne peut donner lieu à remboursement. Cet exemple, on l'espère, restera exceptionnel. Il témoigne néanmoins que ce qui peut apparaître comme une avancée majeure dans les premières parties du dossier standard de demande d' AMM $^{14}$ relatives à la documentation chimique, pharmaceutique, biologique et biotechnologique (module 3) n'est pas toujours corrélée à un bénéfice pour le malade, d'après les données qui figurent dans le module consacré à la documentation clinique (module 5).

\section{Des problèmes éthiques nouveaux et originaux?}

Les questions éthiques abordées sont liées à l'utilisation d'éléments ordinaires du corps humain, excluant deux cas particuliers, celui des cellules germinales et celui des cellules souches embryonnaires. Ces derniers éléments concernent la transmission de la vie et le respect du droit à la vie. Aussi, posent-ils des questions spécifiques qui ont précisément eu pour effet de retarder la rédaction et l'adoption du règlement de novembre 2007.

La particularité de ces nouveaux médicaments découle de leur origine et du glissement opéré, à la fois dans l'ordre des choses et dans l'ordre juridique. Les opérations réalisées transforment des éléments ou produits du corps humain en produits de santé, en médicaments qui seront utilisés, consommés par le même patient, par un autre patient ou par un groupe de patients. Or, tout médicament est un produit marchand, régi par toutes les règles du marché : recherche de profit, logique consumériste, concurrence, promotion, publicité... Ce passage signifie donc une rupture, puisque l'abandon du statut 
d'élément ou produit du corps humain amène la suppression des garde-fous éthiques et des principes du bio-droit destinés à sauvegarder la dignité de la personne et à protéger la personne dans son corps en préservant notamment celui-ci de la commercialisation. Le corps humain peut alors apparaître comme une mine au sein de laquelle on va puiser du matériel qui, moyennant des opérations plus ou moins complexes, sera transformé et utilisé comme un produit de santé. Dès lors que la perméabilité entre les deux types de produits, produit du corps humain et produit de santé, est acquise, jusqu'où va-t-on aller dans la manipulation de la structure même du vivant, dans la maîtrise du corps humain, voire dans la reconstruction de l'homme ? Comment alors distinguer les frontières de l'humain et continuer à reconnaître et respecter la dignité de la personne humaine pour la traiter toujours comme une fin en soi et jamais comme un moyen, alors même que le corps d'une personne vivante devient un réservoir de médicaments en puissance? Comment ne pas y voir un danger de réification, puisqu'en paraphrasant l'expression commune relative au double diagnostic préimplantatoire, on pourrait parler d'homme médicament ${ }^{15}$ ? ${ }^{16}$ Comment éviter la prévalence des intérêts commerciaux et la logique de profit si ces médicaments sont fabriqués à large échelle par des entreprises industrielles ? Le socle des principes fondamentaux -dignité de la personne, inviolabilité, indisponibilité et non-patrimonialité du corps humain- pourrait être profondément ébranlé.

Cependant il ne s'agit pas ici de problèmes éthiques nouveaux ${ }^{17}$. Il suffit d'étendre à ces nouveaux médicaments biologiques les mesures introduites précédemment pour des produits simi- laires, notamment les produits stables dérivés du sang qui sont des médicaments. En effet, il faut d'une part souligner que les pratiques relatives aux MTI sont en général bien moins invasives, massives et spectaculaires que les transfusions sanguines ou que les greffes d'organes, autorisées dans nos pays depuis des décennies; et que d'autre part, les règles relatives à l'information et au consentement du donneur, à l'anonymat et à la gratuité du don d'éléments ou de produits du corps humain ont permis de sauvegarder les principes qui garantissent le respect de la personne dans son corps. Ceci se vérifie aussi bien au niveau européen, avec notamment la directive de 1989 relative aux produits stables dérivés du sang $^{18}$ et la directive relative à l'établissement des normes de qualité et de sécurité pour le don, l'obtention, le contrôle, la transformation, la conservation, le stockage, et la distribution des tissus et cellules humaines de $2004^{19}$, qu'au niveau français avec la transposition de ces textes dans le CSP et avec les dispositions introduites dans les premiers articles du code civil par la loi relative à la bioéthique du 29 juillet 1994 .

\section{Les spécificités souvent oubliées}

D'autres particularités ou originalités de ces produits de thérapie génique ou cellulaire ou d'ingénierie tissulaire, liées au corpus du droit pharmaceutique, ont été moins commentées.

\section{Particularités du règlement européen concer- nant les MTI}

\section{Le rôle pionnier de l'Union européenne}

Il est intéressant de mettre en évidence l'avance 
de l'UE sur les États-Unis qui lui servent habituellement de modèle. Alors que 1'UE n'avait fait que suivre et copier, respectivement dix-sept et huit années plus tard, les initiatives nord-américaines en introduisant des régimes particuliers pour les médicaments orphelins ${ }^{20}$ d'une part et pour les médicaments pédiatriques ${ }^{21} \mathrm{~d}$ 'autre part, elle a été la première à instaurer un statut propre aux MTI et à y associer des règles spécifiques. Il est encore plus intéressant et étonnant de voir que les États-Unis ne se sont toujours pas dotés d'un régime particulier pour ces médicaments. Pourquoi cette singulière avance qui contraste avec le retard mentionné précédemment? Le recul des années - cinq ans déjà - nous permet de confirmer que l'UE n'a pas été à proprement parler précurseur ou pionner puisqu'elle n'a pas encore été suivie par d'autres pays. Pourquoi une telle anticipation et quel est son effet ? Le nombre d'AMM délivrées reste dérisoire; était-ce prévu initialement? Les espérances ontelles été déçues parce qu'exagérées ? D'autres mesures auraient-elles dû être mises en œuvre pour insuffler une puissante dynamique aux parties prenantes ? L'histoire du règlement et son incidence devront être approfondies ${ }^{22}$.

Mais d'ores et déjà, il convient de s'arrêter sur le choix, significatif, du règlement pour cette lex specialis, instrument encore rarement utilisé dans la législation pharmaceutique communautaire de l'époque. En choisissant l'effet immédiat et en évitant tout risque de manque d'harmonisation dans l'interprétation des règles entre États membres (EM), l'acte juridique de 2007 constitue un jalon dans la politique pharmaceutique de l'UE en faveur de l'innovation. C'est un message fort à l'attention des acteurs du secteur, un appel à dyna- miser la recherche et à réduire le retard des firmes européennes par rapport à leurs concurrentes états-uniennes ou japonaises. Cette initiative européenne s'inscrit dans la «stratégie relative aux sciences du vivant et de la biotechnologie $»^{23}$, dans la droite ligne des orientations tracées par le Conseil européen de Lisbonne en mars 2000 qui ambitionnait de faire de l'UE «l'économie de la connaissance la plus compétitive et la plus dynamique du monde ». L'antériorité de l'UE semble ainsi résulter de cette volonté d'émulation pour les chercheurs et tous les acteurs du secteur pharmaceutique des États-membres (EM).

\section{Le poids des considérations économiques}

Le règlement de 2007 est né de la prise en compte de diverses considérations économiques, ellesmêmes étroitement intriquées. Si le texte affiche explicitement comme "objectif essentiel la sauvegarde de la santé publique $»^{24}$, la base juridique marché intérieur, indéniablement, s'avère également première ${ }^{25}$. La stratégie d'innovation et la stratégie de compétitivité sont tellement inséparables qu'on se demande laquelle est première. Il est donc difficile de distinguer tous ces objectifs économiques : promouvoir le marché des MTI, assurer la libre circulation de ces nouveaux produits au sein de l'UE en éliminant tous les obstacles qui pourraient constituer un frein aux échanges entre EM, et plus largement assurer le bon fonctionnement du marché intérieur dans le secteur des biotechnologies, tout en favorisant la compétitivité des entreprises européennes. Un des sept chapitres du règlement est précisément consacré aux mesures incitatives, principalement l'accessibilité de l'expertise de l'Agence européenne du médicament (EMA) tout au long du 
développement du médicament et la réduction des redevances. Des mesures particulières sont prises pour les petites et moyennes entreprises qui investissent dans la recherche de ces médicaments : avantages en matière de développement et avantages fiscaux.

\section{Pluralité des régimes juridiques associés aux thérapies innovantes}

\section{La mosaïque des statuts}

La spécificité qui nous apparaît la plus marquante réside dans la multiplicité des statuts des produits et de leur régime juridique, notamment sur le plan institutionnel ${ }^{26}$. Nous avons parlé jusqu'ici des MTI dont l'AMM ne peut être octroyée que par l'EMA selon une procédure centralisée qui fait intervenir un comité créé $a d$ hoc par le règlement de 2007, le Comité des thérapies innovantes. Mais le règlement a par ailleurs prévu une exemption hospitalière, pour des MTI préparés ponctuellement (MTI-PP), qui répondent bien aux conditions de la définition du médicament mais sont sous tutelle de l'autorité nationale.

En France, il sont fabriqués moyennant l'autorisation de l'Agence nationale de sécurité du médicament et des produits de santé (ANSM), selon des normes de qualité spécifiques, à l'intention d'un malade déterminé, et utilisés dans un hôpital, sous la responsabilité d'un médecin, pour exécuter une prescription médicale déterminée ${ }^{27}$. Ils ne peuvent être préparés que sur le territoire national soit par des établissements pharmaceutiques, soit, par dérogation au monopole pharmaceutique, par des établissements autorisés par ANSM. L'organigramme de l'ANSM leur accorde une belle place puisque l'une des huit Directions Produits s'intitule Direction des thérapies innovantes, des produits issus du corps humain et des vaccins ${ }^{28}$.

À côté de ces deux grands groupes de médicaments, il y a encore un autre statut de produits de santé qui eux ne constituent pas des médicaments : les préparations, produits cellulaire ou tissulaire, allogéniques ou autologues, à finalité thérapeutique ; exemple, les cellules souches hématopoḯtiques indiquées pour la reconstitution hématopoïétique et immunitaire. Deux critères permettent de les distinguer des MTI-PP : l'existence de modifications substantielles des cellules ou tissus et/ou le changement de la fonction essentielle des cellules ou tissus, deux éléments constitutifs des définitions respectivement de médicaments de thérapie cellulaire et d'ingénierie cellulaire ou tissulaire. Ces produits relèvent de la compétence nationale et sont réglementés dans chaque EM sur la base de la directive 2004/23/ CE précitée. Ne s'agissant pas de médicaments, ils ne sont bien sûr pas réservés au monopole pharmaceutique.

Ces trois catégories peuvent donner lieu à deux typologies : la première, évidente, oppose les médicaments aux préparations. Traditionnellement, médicament implique, surtout en France, monopole pharmaceutique de fabrication, de distribution en gros et de dispensation, avec la garantie de la qualité des actes pharmaceutiques apportée par la présence d'un pharmacien responsable tout au long de la chaîne. Mais ce lien connaissant de plus en plus de dérogations, y compris pour les MTI et MTI-PP, on ne peut pas établir de dichotomie sur ce critère entre MTI et préparations à finalité thérapeutique. 
La seconde partition oppose les modes de fabrication et reprend la distinction entre médicaments préparés industriellement et médicaments fabriqués de manière artisanale : «L'un des traits essentiels de l'objet industriel étant la plus exacte uniformité de production, l'interchangeabilité parfaite, c'est un lieu commun de noter que l'une des conditions de l'industrie au moins dans sa phase naissante et dans la phase contemporaine, est une lutte contre l'individuation. ${ }^{29}$. Préparation industrielle signifie en effet capacité de reproduire la formule à l'identique, avec une variabilité entre deux unités produites qui tend vers zéro, ce qui est recherché par un système d'assurance qualité qui prévoit des contrôles systématiques. Ce haut degré d'exigence de qualité pharmaceutique qui s'impose aux spécialités pharmaceutiques et aux MTI ne peut être égalé pour les produits qui ne sont pas fabriqués industriellement.

Deux remarques sur cette industrialisation :

- ce qui freine le développement industriel des MTI ne se situe pas tant au niveau des process de fabrication à mettre en place à large échelle mais bien plutôt en amont, dans la conception de formules valables pour tous les patients, compte tenu de la singularité des cas cliniques et des risques de problèmes immunologiques notamment ;

- rien ne s'oppose en théorie à ce que les préparations à finalité thérapeutique soient fabriquées à l'échelle industrielle; elles resteraient alors de la compétence nationale.

Ces deux critères distinctifs n'apparaissent donc pas concluants. En revanche, ce qui semble vraiment discriminant, c'est l'obligation d'obtenir une AMM ; en l'occurrence, pour les seuls MTI, et auprès de l'EMA. Là se situe le véritable clivage. Car l'octroi de l'AMM est la caution la plus élevée des pouvoirs publics pour proposer aux patients un produit conforme à la formule annoncée et dont le rapport bénéfice/risque a été évalué selon des méthodes scientifiques rigoureuses et éprouvées. Il résulte d'une procédure d'expertise contradictoire destinée à apprécier le caractère satisfaisant des niveaux de preuve des données apportées par la firme en matière de qualité, d'innocuité et d'efficacité. Pour toutes ces raisons, le législateur français n'autorise l'utilisation de préparations artisanales, préparations magistrales et hospitalières, qu'en cas d'absence de spécialité pharmaceutique autorisée ${ }^{30}$.

\section{Les enjeux de ces classifications}

La multiplicité des catégories de produits répond à la variété des éléments du corps humain et à la grande diversité des technologies employées. Le législateur a tenté de moduler les exigences en matière de règles relatives à la recherche, aux établissements, aux produits, aux procédés, en fonction des risques potentiels en vue de garantir la sécurité sanitaire. En matière d'autorité de tutelle, la dichotomie niveau européen/niveau national ne pose pas de problème en France, compte tenu du haut niveau de culture d'évaluation médico-administrative ; il est en revanche probable que d'autres pays moins fortunés et moins avancés sur le plan scientifique ne disposent pas d'experts spécialisés dans ces domaines de pointe pour assurer aussi bien que l'EMA les fonctions dévolues. Des écarts entre EM sont ainsi prévisibles. 
En pratique, les acteurs du secteur embarrassés à déterminer le statut de leur produit sont invités à se rapprocher des autorités compétentes. En effet, les ambiguités peuvent se présenter puisque deux produits préparés de manière identique peuvent être associés à deux statuts distincts en fonction de leur mode d'utilisation ou de leur finalité, et plus généralement, à une multitude de statuts correspond un grand périmètre de frontières et donc une large gamme de produits frontières. Pour éviter des erreurs lourdes de conséquences pour les promoteurs, l'ANSM a publié un guide ${ }^{31}$. Il conviendra dans quelques années de vérifier l'appréhension des frontières par les acteurs du secteur et la justesse des choix stratégiques réalisés dès les premières étapes de développement des produits.

Pour conclure, revenons sur l'un des objectifs du règlement de 2007 : remédier aux inégalités. La norme de droit a permis de clarifier le cadre juridique applicable à ces nouveaux produits et d'harmoniser ces règles pour l'ensemble des vingt-sept EM afin d'en faciliter l'accès. En dépit de variations d'application des règles dans le champ laissé à la compétence nationale, les législateurs européen et français ont atteint leur but ; en tout cas, ils ne pouvaient probablement pas faire davantage.

Mais la règle de droit se révèle bien impuissante pour permettre d'accélérer le processus de développement des médicaments. Le monde de la recherche a ses propres lois indépendamment des normes juridiques, et le progrès ne se construit pas uniquement dans les assemblées parlementaires. La règle de droit se révèle tout aussi inefficace pour obtenir des financements. Et le facteur limitant le plus fort pour freiner l'accessibilité de ces produits tant attendus ${ }^{32}$ est indéniablement la capacité à trouver les ressources de ce financement socialisé, tant en France, qu'au niveau européen - pays du Sud, pays de l'Est -, qu'a fortiori au niveau planétaire. 
$\mathrm{N}$

1. E. Fouassier, "Médicament", Droit pharmaceutique, Lexis-Nexis, 2011, 4.

2. J. Peigne, "Le nouveau statut des produits de thérapie génique et cellulaire." Revue de Droit sanitaire et social $n^{\circ} 2 / 2005: 220-231$.

3. La loi du 28 mai 1996 avait introduit une classification des cellules selon leur milieu de prélèvement et leur finalité instaurant notamment la catégorie des produits biologiques à effet thérapeutique. La loi du 6 août 2004 relative à la bioéthique avait supprimé cette classification. Notons cependant que dès 1995 existait à l'Agence du médicament une commission de thérapie génique.

4. Règlement (CE) n 1394/2007 du Parlement européen et du Conseil du 13 novembre 2007 concernant les médicaments de thérapie innovante et modifiant la directive 2001/83/CE ainsi que le règlement (CE) $n^{\circ} 726 / 2004$.

5. Préparations réalisées à l'échelle industrielle versus préparations artisanales.

6. Médicaments immunologiques, radiopharmaceutiques, dérivés du sang, biologiques et biologiques similaires.

7. Médicaments à prescription facultative versus médicaments à prescription obligatoire au sein desquels il faut encore différencier les médicaments sur prescription médicale, renouvelable ou non renouvelable, les médicaments soumis à prescription médicale spéciale (stupéfiants) et les médicaments soumis à prescription médicale restreinte. Il faut encore rajouter d'autres catégories propres au droit français. 8. Ordonnance du 26 avril 2007 (JO du 27-4-07), modifiant l'article L. 5121-1 CSP.

9. J. Peigne, "Le droit des biothérapies : entre subsidiarité éthique et harmonisation technique", Revue de
Droit sanitaire et social n²/2008 : 292-306.

10. Cité par J. Ménard, P. Graain-Kibleur, "La politique de l'innovation en santé". Actualité et dossier en santé publique, 2002, 39, p. 18.

11. ChondroCelect 10000 cellules/microlitre, suspension pour implantation. Laboratoire TIGENIX NV.

Cellules de cartilage autologues viables, caractérisées, amplifiées ex vivo exprimant des protéines marqueurs spécifiques.

12. Avis du 6 octobre 2006. Disponible sur le site de la Haute autorité de santé.

13. Conformément à l'article R. 163-3 CSS.

14. La présentation du dossier que doit fournir le laboratoire aux autorités de tutelle est harmonisée au niveau communautaire, il s'agit du Common Technical Document.

15. R. Andorno, "Le diagnostic préimplantatoire dans les législations des pays européens: sommes-nous sur une pente glissante ?" Bioethica Forum / 2008 / Volume $1 / \mathrm{n}^{\circ} 2: 96-103$

16. Voir Infra. Etude DPI-HLA.

17. E. Fouassier, Médicament. Droit pharmaceutique. Lexis-Nexis. $2011: 4, n^{\circ} 61$ et 62 .

18. Directive 89/381/CEE du 14 juin 1989 (JOCE du 28 juin 1989) concernant le rapprochement des dispositions législatives, réglementaires et administratives relatives aux spécialités pharmaceutiques, transposée en France par la loi du 4 janvier 1993.

19. Directive 2004/23/CE du Parlement européen et du Conseil du 31 mars 2004.

20. Alors que c'est dès 1983 que les États-Unis ont adopté la loi Orphan Drug Act pour inciter les laboratoires pharmaceutiques à investir dans la recherche de médicaments destinés à des maladies orphelines, suivis par le Japon en 1993, c'est seulement en 2000 que l'UE a introduit une législation équivalente (Règl. (CE) n 141/2000 du Parlement européen et du Conseil du 16 décembre 1999 concernant les médicaments or- 
phelins et Règl. (CE) n ${ }^{\circ}$ 847/2000 de la Commission du 27 avril 2000 établissant les dispositions d'application des critères de désignation d'un médicament en tant que médicament orphelin et définissant les concepts de « médicament similaire » et de « supériorité clinique »). 21. C'est dès 1998 qu'aux États-Unis une règlementation spécifique a incité les laboratoires à développer des médicaments pédiatriques ; l'UE a introduit une législation équivalente avec le Règl. (CE) n 1901/2006 du Parlement européen et du Conseil du 12 décembre 2006 relatif aux médicaments à usage pédiatrique.

22. Voir Infra. Nathalie Schiffino, "Les thérapies innovantes au prisme de l'évaluation des politiques publiques et de l'appréhension du risque".

23. $\operatorname{COM}(2002) 27$ du 23 janvier 2002 et M. Blanquet, "La vision stratégique européenne des biotechnologies et des OGM", in Sécurité alimentaire et OGM, R. Quellet (dir), Presses de l'Université des sciences sociales de Toulouse, $2007: 15$.

24. Deuxième considérant.

25. M. Blanquet et N. De Grove-Valdeyron, "Les enjeux et les apports du règlement communautaire concernant les médicaments de thérapie innovante". Revue des Affaires Européennes 2006/4 : 689-721.

26. A. Mahalatchimy, E. Rial-Sebbag, V. Tournay, A. Faulkner, "The legal landscape for Advanced Therapy Medicinal Products: material and institutional implementation of European Union rules", Journal of Law and Society, Volume 39, $\mathrm{N}^{\circ} 1$, March 2012, pp.131-149, ainsi que E. Cadeau. J.-Y. Richeux, "Produits et préparations de thérapies cellulaire et génique - Statut juridique". Droit pharmaceutique. Lexis-Nexis. $2011:$ 61-70.

27. Article L. 5121-1 CSP, $17^{\circ}$.

28. Cette direction est compétente en tant qu'interlocuteur de l'EMA pour les MTI, et est directement concernée pour les MTI-PP et les préparations.
29. G. Granger Essai d'une philosophie du style, Paris. Armand Colin. 1968, p. 9.

30. Article L. 5121-2 CSP.

31. ANSM. MTI, MTI-PP et préparations : Synthèse du cadre réglementaire applicable pour la fabrication, le développement et la mise sur le marché de ces produits. Version 1 du 30 mars 2012. Mise à jour 5 juin 2012. Disponible sur le site.

32. A. Mahalatchimy, "Access to advanced therapy medicinal products in the EU: where do we stand ?" European Journal of Health Law, $\mathrm{n}^{\circ} 18,2011$, pp. 305-317, ainsi que A. Mahalatchimy, "L'harmonisation de l'accès au marché des médicaments de thérapie innovante : entre volonté et réalité", Revue Générale de Droit Médical, $\mathrm{n}^{\circ}$ 33, décembre 2009, pp. 257- 272. 


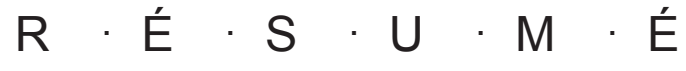

La thérapie génique, la thérapie cellulaire, l'ingénierie tissulaire constituent de nouvelles avancées dans la maîtrise du corps humain, ou, diront certains, dans la manipulation de la structure même du vivant et dans la reconstruction de 1'homme. Celui-ci n'est plus seulement l'auteur de la technique, il en devient aussi l'objet, le résultat. C'est sans doute parce qu'un saut ontologique a été perçu que la législation européenne a créé en 2007 de nouvelles catégories de médicaments, sous le nom générique de «médicaments de thérapie innovante » (règlement $\left.n^{\circ} 1394 / 2007\right)$.

Il est intéressant de souligner qu'alors que l'Union européenne n'avait fait que suivre les initiatives des États-Unis et du Japon en introduisant des régimes particuliers pour les médicaments orphelins d'une part et pour les médicaments pédiatriques d'autre part, elle a été la première à instaurer un statut particulier aux médicaments de thérapie innovante et à y associer des règles spécifiques. L'objectif affiché était double : à la fois promouvoir leur marché et garantir un niveau élevé de protection de la santé publique. C'est tout le dispositif d'évaluation qui est renforcé, les procédures d'enregistrement faisant appel à une nouvelle instance ad hoc.

Quel est le fondement de ces dérogations et de toutes ces exigences supplémentaires ? En effet, ces produits ne sont pas les premiers médicaments d'origine humaine. Depuis la nuit des temps, l'arsenal thérapeutique comprend des médicaments fabriqués à partir d'éléments du corps humain, et de fait, depuis quelques décennies, l'ensemble des «biothérapies » explose. L'analyse du droit européen et du droit français relatif à ces différentes catégories de biens marchands permettra de distinguer ce qui relève de la continuité de ce qui constitue d'éventuelles ruptures. Ainsi, au regard des processus de fabrication de ces médicaments, on s'interrogera sur les spécificités de leur encadrement en démontrant qu'il s'agit de simples différences de degré et non de différences de nature.

\section{Abstract \\ Advanced therapy medicinal products : which specific features in pharmaceutical law?}

Gene therapy, cell therapy and tissue engineering constitute new advances regarding the control of the human body, or as some could say, regarding the manipulation of the living structure and the reconstruction of the human person. The latter is not only the technique's creator; he becomes also its object, its result. The perception of an ontological step is probably the reason why a new legal category of drugs called "advanced therapy medicinal products" has been established in 2007 by the EU legislation (Regulation (EC) $\left.n^{\circ} 1394 / 2007\right)$.

It is interesting to highlight the European Union just followed the American and Japanese initiatives by setting-up specific legal regimes for orphan and paediatric drugs. However, it has been the first to adopt a particular statute and associated rules for advanced therapy medicinal products. There was a double objective displayed: to promote their market and to ensure a high level of human health protection. The whole assessment process has been strengthened, a new ad hoc committee being involved in the registration procedures.

What is the basis for these exceptions and for all these supplementary requirements? These products are not the first drugs based on human body elements. For a 
long time, they have been part of the therapeutic package. Consequently, the whole "biotherapies" is exploding for few decades. The analysis of the European and French laws regarding these different categories of market goods permits to distinguish what constitutes a continuum from what constitutes potential breaches. Thus, regarding the development processes of drugs we will questioned the specificities of their legal frame by proving they are only degrees differences and not nature differences 
